\title{
High resolution tumor targeting in living mice by means of multispectral optoacoustic tomography
}

Andreas Buehler ${ }^{1}$, Eva Herzog ${ }^{1}$, Angelica Ale ${ }^{1}$, Bradley D Smith², Vasilis Ntziachristos ${ }^{1}$ and Daniel Razansky ${ }^{1 *}$

\begin{abstract}
Background: Tumor targeting is of high clinical and biological relevance, and major efforts have been made to develop molecular imaging technologies for visualization of the disease markers in tissue. Of particular interest is apoptosis which has a profound role within tumor development and has significant effect on cancer malignancy.

Methods: Herein, we report on targeting of phosphatidylserine-exposing cells within live tumor allograft models using a synthetic near infrared zinc(II)-dipicolylamine probe. Visualization of the probe biodistribution is performed with whole body multispectral optoacoustic tomography (MSOT) system and subsequently compared to results attained by planar and tomographic fluorescence imaging systems.

Results: Compared to whole body optical visualization methods, MSOT attains remarkably better imaging capacity by delivering high-resolution scans of both disease morphology and molecular function in real time. Enhanced resolution of MSOT clearly showed that the probe mainly localizes in the vessels surrounding the tumor, suggesting that its tumor selectivity is gained by targeting the phosphatidylserine exposed on the surface of tumor vessels.
\end{abstract}

Conclusions: The current study demonstrates the high potential of MSOT to broadly impact the fields of tumor diagnostics and preclinical drug development.

Keywords: Optoacoustic imaging, Tumor targeting, Molecular imaging, Phosphatidylserine targeting

\section{Background}

A versatile pool of optical reporter agents and imaging methods for enhancement and probing of anatomical features and molecular function of tumors has been recently developed $[1,2]$. The contrast carriers reach from fluorochromes [3] and other organic dyes to quantum dots [4] and several forms of nanoparticles [5,6] with a large selection of accumulation, targeting and activation mechanisms $[7,8]$. It has been long realized that apoptosis plays a profound role within tumor development and has significant effect on cancer malignancy [9]. Therefore, many anticancer treatment strategies are based on the induction of apoptosis in tumors [10], and early monitoring of such treatment success is of great interest within preclinical drug development. Even though optical molecular markers of apoptosis are

\footnotetext{
* Correspondence: dr@tum.de

'Institute for Biological and Medical Imaging, Technische Universität München und Helmholtz Zentrum München, Ingoldstädter Landstraße 1, Neuherberg 85764, Germany

Full list of author information is available at the end of the article
}

generally available [11,12], their in vivo visualization is challenging due to intensive light scattering in living tissues. Some earlier attempts to specifically image apoptotic responses in whole living animals using fluorescence molecular tomography attained generally low spatial resolution and imaging speed $[13,14]$. As a result, questions related to the exact probe localization (e.g., necrotic foci within the tumors versus surface of the tumor blood vessels) cannot be answered using those methods.

More recently, multispectral optoacoustic tomography (MSOT) has been emerging as a promising tool for high-resolution volumetric imaging of optical contrast in tissues [15], capable of visualizing tissue chromophores with ultrasonic resolution independent from light scattering [16]. The use of multispectral methods allows to efficiently resolve extrinsic optical agents, such as fluorochromes and fluorescent proteins [17,18]. Optoacoustic imaging correspondingly offers important advantages in small animal imaging, including the use of non-ionizing radiation, the versatile sensing of chromophoric 
molecules for probing cellular and sub-cellular function, good spatial resolution and, in analogy to ultrasound imaging, real-time operation [19-21].

Recently, a novel optical probe, comprised of a synthetic zinc(II)-dipicolylamine complex appended to a near-IR (NIR) carbocyanine fluorophore (subsequently called PSS-794), has been shown to selectively target anionic membrane-bound phosphatidylserine (PS) exposed by dead and dying cells within xenograft tumors in rat and mouse models [22]. Usually restricted to the inner leaflet of the plasma membrane, phosphatidylserine is selectively exposed as an early event during cell apoptosis, which is a crucial mechanism of cell number control in various physiological and pathological events [23]. Herein, we investigate whether PSS-794 can be used to image tumors in vivo with high resolution using MSOT.

\section{Methods}

Multispectral optoacoustic tomography system

A real-time MSOT scanner, similar to the one described by Razansky et al. [21], was utilized in this study (Figure

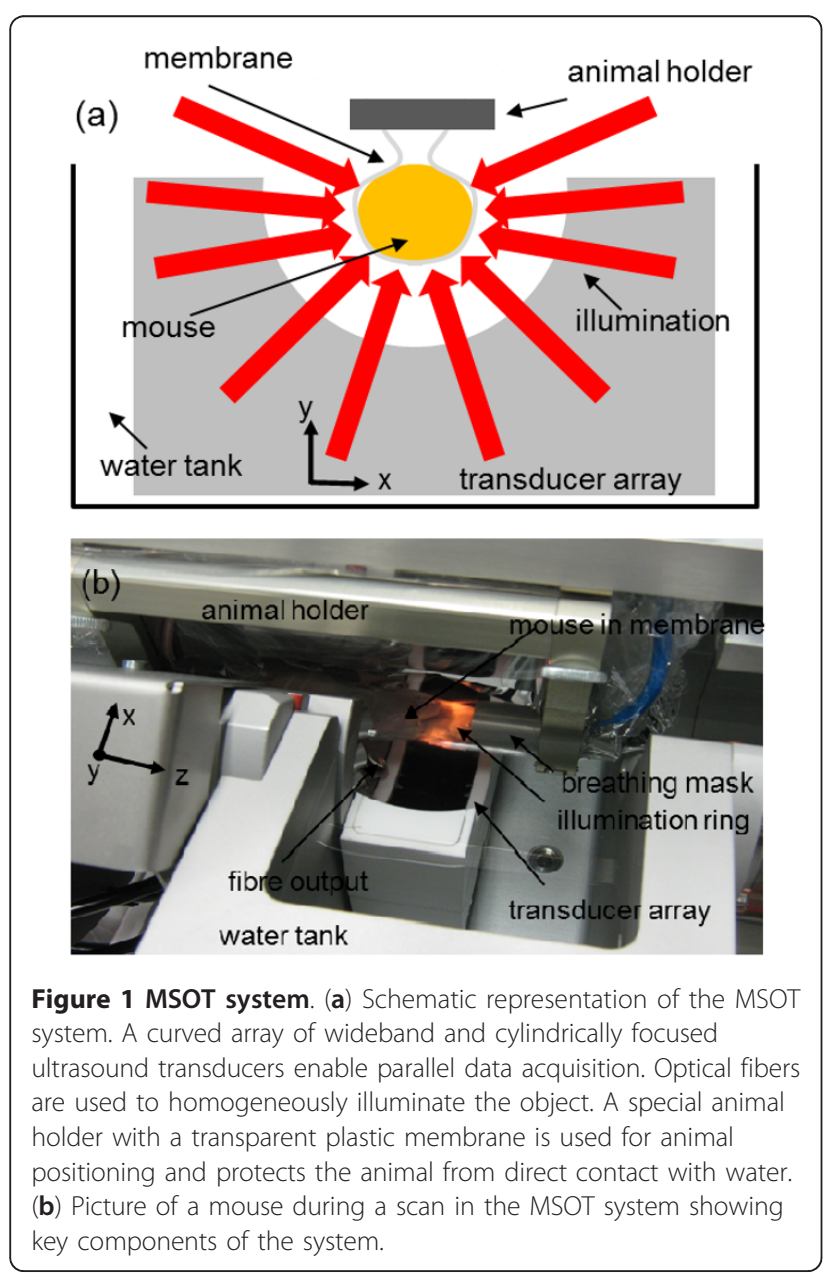

1). Briefly, it is based on an excitation source from a tuneable (680-950 nm) optical parametric oscillator laser (Phocus, Opotek Inc., Carlsbad, CA, USA) delivering < 10-ns duration pulses with repetition frequency of 10 $\mathrm{Hz}$. The laser beam is guided into a silica fused-end fiber bundle (PowerLightGuide, CeramOptec $\mathrm{GmbH}$, Bonn, Germany) consisting of 630 fibers partitioned into ten arms, thus, creating a ring-shaped illumination pattern of approximately 8-mm width upon the surface of the animal. The beam was sufficiently broadened to keep the laser pulse fluence on the surface of imaged objects under $20 \mathrm{~mJ} / \mathrm{cm}^{2}$ in order to meet the laser safety standards. Signal collection is based on a custommade 64-element focused transducer array (Imasonic $\mathrm{SaS}$, Voray, France) covering a solid angle of $172^{\circ}$ around the imaged object, while the detection plane coincided with the center of the illumination ring. The individual detection elements are manufactured using piezocomposite technology with central frequency of 5 $\mathrm{MHz}$, bandwidth of more than $50 \%$, and sensitivity of approximately $18 \mu \mathrm{V} / \mathrm{Pa}$ and are shaped to create a cylindrical focus at $40 \mathrm{~mm}$. According to ultrasonic diffraction limit and focal width at the central frequency, the effective spatial resolution of the system is estimated at $150 \mu \mathrm{m}$ (in-plane) and $800 \mu \mathrm{m}$ (elevational). The detected signals are digitized at 60 megasamples per second frequency by eight multichannel analog to digital converters (PXI5105, National Instruments, Austin, TX, USA) with noise floor of approximately $3.8 \mathrm{nV} / \sqrt{\mathrm{Hz}}$. To facilitate in vivo measurements, the animals were wrapped by a water-impenetrable transparent membrane that averts animal contact with water inside the imaging chamber. A linear stage (NRT150, Thorlabs GmbH, Karlsfeld, Germany) allows linear translation of the animal holder in the axial $z$ direction for the acquisition of three-dimensional data sets.

\section{Animal imaging}

All procedures involving animals and their care were conducted in full agreement with the institutional guidelines, complying with national and international laws and regulations. For imaging, four female 8-week old athymic CD-1 nude mice containing 4T1 tumor allografts were used. The tumors were obtained by injecting $0.8 \times 10^{5} 4 \mathrm{~T} 1$ cells subcutaneously into the mouse's neck where they were allowed to grow for 7 days, reaching a diameter of 0.4-0.5 cm. The PSS-794 imaging probe was prepared as previously reported [24]. The first two tumor-carrying mice were injected intravenously via the tail vein $24 \mathrm{~h}$ prior to imaging with 6 $\mathrm{mg} / \mathrm{kg}$ (100 nmoles) and $3 \mathrm{mg} / \mathrm{kg}(50 \mathrm{nmol})$ of the probe, respectively. A third mouse was injected $3 \mathrm{~h}$ prior imaging with probe amount of $3 \mathrm{mg} / \mathrm{kg}(50 \mathrm{nmol})$. 
For control, a fourth mouse was injected with $3 \mathrm{mg} / \mathrm{kg}$ (50 nmol) of indocyanine green (ICG), a non-targeted dye having spectral characteristics similar to PSS-794 in the near-infrared, $3 \mathrm{~h}$ prior imaging [21]. The mice were anesthetized with a mixture of ketamine and xylazine, and were placed in supine position in the animal holder. Cross-sectional multispectral optoacoustic image datasets were acquired through the tumor at six different wavelengths in the NIR window (700, 740, 760, 780, 800 , and $900 \mathrm{~nm}$ ). For quantitative readings, the interwavelength laser energy variations were corrected for by normalizing the data by power meter readings (FieldMaxII-TOP, Coherent GmbH, Dieburg, Germany).

\section{Image reconstruction and analysis}

Reconstruction of single-wavelength optoacoustic images was done with interpolated matrix model inversion method [25]. Prior to inversion, the raw optoacoustic signals were bandpass-filtered between $50 \mathrm{kHz}$ and 7 $\mathrm{MHz}$, and deconvolved with the combined electrical impulse response of the ultrasonic detectors and the acquisition system [26]. For inversion of the forward matrix, the iterative PLSQR algorithm has been used [27]. To selectively detect the biodistribution of the probe over intrinsic tissue absorption background, multispectral un-mixing by independent component analysis (ICA) [28] was applied.

This technique is based on the assumption that the source components of the mixed multispectral dataset are statistically independent. In order to identify these source components, the algorithm seeks a transformation of the dependant multispectral dataset into a set of independent variables which is accomplished by maximizing their non-Gaussianity because, according to the central limit-theorem, statistical independent variables are less Gaussian than their mixed dependent counterparts. The measured absorption spectra of the probe and oxygenized and deoxygenized blood were fed to the algorithm as a starting point for a guided un-mixing. The probe component was identified by comparing the resulting spectra from the un-mixing algorithm with the measured probe spectra. In contrast to other methods based on least-square un-mixing, which try to fit an $a$ priori known spectra to the data, the ICA algorithm has more degrees of freedom which reduces the cross talk and leads to generally better performance.

\section{Cross-validation with fluorescence measurements}

For validation purposes, one mouse was also imaged $e x$ vivo with a newly developed $360^{\circ}$ free-space FMT-XCT system which combines fluorescence molecular tomography (FMT) with small animal X-ray CT (XCT) into a hybrid imaging device [29]. The system was capable of simultaneous three-dimensional visualization of small animal anatomy and biodistribution of fluorescent probes in vivo. For volumetric FMT reconstructions, 12 angular projections distributed over full $360^{\circ}$ range were acquired close to the excitation and emission wavelengths of PSS-794. Following the FMT-XCT measurement, the euthanized mice were cooled to $-80^{\circ} \mathrm{C}$. For further verification of the in vivo MSOT and FMT images, cross-sectional color photographs (RGB) and fluorescence images were also obtained using a cryotome supplemented by a home-built multispectral epifluorescence system [30]. The system consisted of a white light source and a sensitive CCD camera with motorized filter wheels for selection of the excitation (740 nm, $40 \mathrm{~nm}$ bandpass filter) and emission $(785 \mathrm{~nm}$ longpass filter) wavelengths.

\section{Histology}

After cryo-slicing, selective tissue samples in the tumor area were used for histological validation for highly specific detection of apoptosis using commercially available annexin V antibody (Abcam PLC, Cambridge, United Kingdom). The appearance of PS residues (normally hidden within the plasma membrane) on the surface of the cell is an early parameter of apoptosis, which can also be used to detect and measure apoptosis. During apoptosis, PS is translocated from the cytoplasmic face of the plasma membrane to the cell surface. Annexin V has a strong Ca2+-dependent affinity for PS and, therefore, can be used as a probe for detecting apoptosis.

\section{Results}

Figure 2 depicts the molar extinction coefficient of the targeted PSS-794 probe in $35 \mathrm{mg} / \mathrm{ml}$ albumin acquired using a fiber optic spectrophotometer (USB2000, Ocean

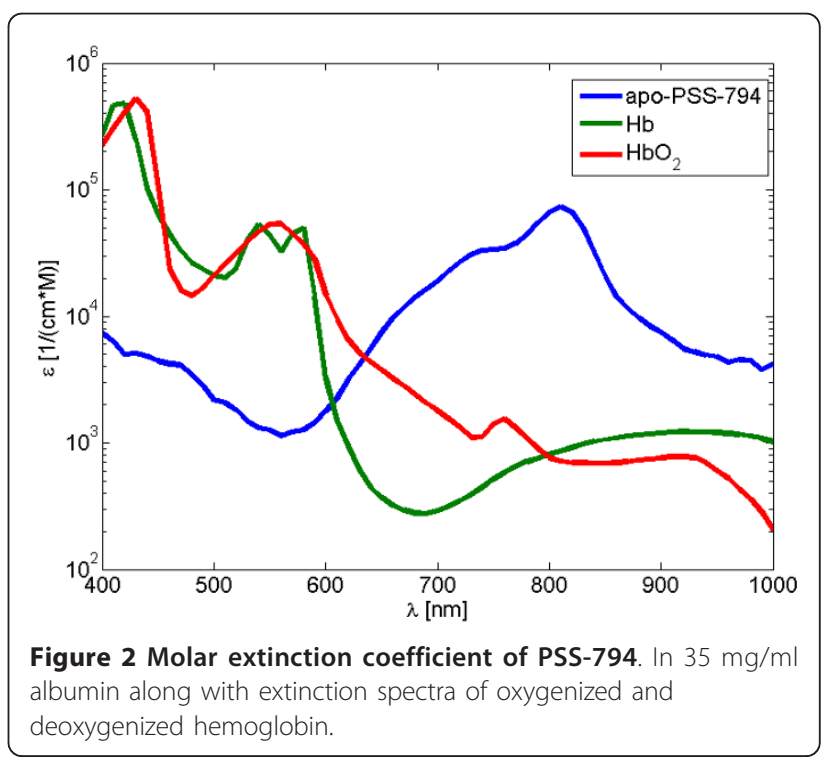


Optics Inc., Dunedin, FL, USA) as well as the spectra of oxygenized and deoxygenized hemoglobin. The spectrum of PSS-794 has a characteristic shape with an absorption maximum at $810 \mathrm{~nm}$ close to the isosbestic point of blood. It, thus, differs significantly from the major background tissue absorbers, making it well suited for multispectral optoacoustic detection with high sensitivity.

Figure 3a, d show the spectrally unmixed MSOT signals from the two mice imaged in vivo $24 \mathrm{~h}$ after injections. The single wavelength optoacoustic images acquired at $900 \mathrm{~nm}$ are shown in gray scale, while the spectrally unmixed MSOT signals are superimposed in color. A transparency threshold of $65 \%$ was applied to the unmixed data when superimposed to the single wavelength background image. The boundary of the tumor is delineated in yellow. Figure 3 h shows the result from the mouse imaged $3 \mathrm{~h}$ after injection, and Figure $3 \mathrm{k}$ the result for the mouse injected with ICG also imaged $3 \mathrm{~h}$ after injection. The PSS-794 signal is visible in all the three cases, whereas signal from ICG is not detectable. Apparently, analysis of the high-resolution MSOT images clearly shows that the PSS-794 probe mainly accumulates in the blood vessels surrounding the tumors, while no infiltration of the probe into the tumor mass occurs.

On the other hand, reconstruction of the subsequent ex vivo FMT measurement, shown in Figure 4c, generally confirms the fact that the probe specifically accumulated around the tumor areas. Yet, due to the lower

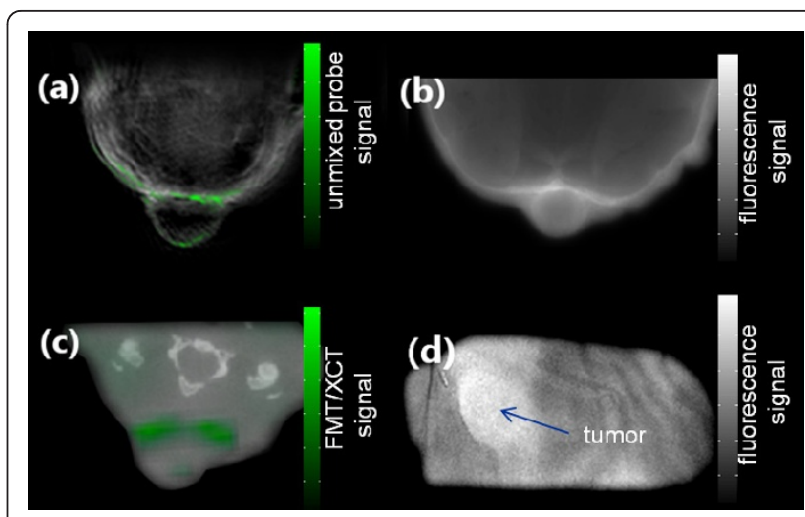

Figure 4 Comparison between optical imaging and MSOT Image (a) shows the MSOT image, and (b) the corresponding epifluoresence image. An FMT-XCT reconstruction is shown in (c) and a planar transillumination image in $(\mathbf{d})$

resolution of the method, it is difficult to determine whether the fluorescence signal is originating only from the vasculature or from within the tumor parenchyma. The exact origin of the fluorescence signal is also not recognizable from the poorly resolved transillumination fluorescence image (Figure $4 \mathrm{~d}$ ). The results of the validation cryo-slicing and epi-fluorescence studies, made on euthanized mice, are shown in Figure 3b, e, i, l. The color pictures of the cryo-sliced mouse in Figure $3 \mathrm{c}, \mathrm{f}, \mathrm{j}$, g shows the actual location of the tumor mass, which can also be readily delineated on the single-wavelength optoacoustic images. Observation of the brown spots in

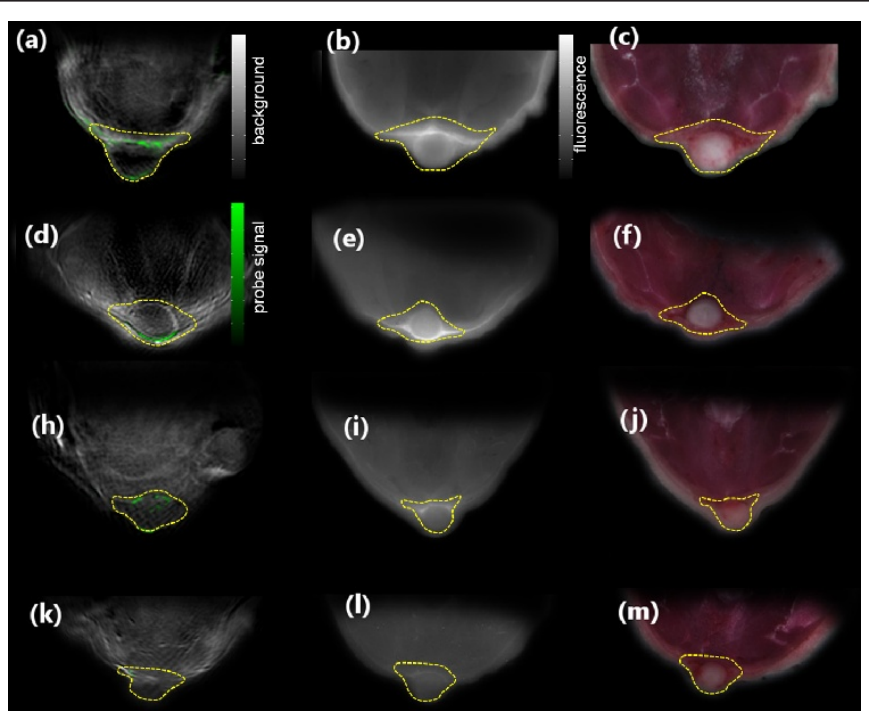

Figure 3 Imaging of targeted apoptotic marker (PSS-794) in 4 T1 tumor-bearing mice. In the first column, the MSOT images are shown. Images (a) and (d) show superposition of a single-wavelength (anatomical) optoacoustic image (in gray scale) and the unmixed component corresponding to the PSS-794 signal in color for the two mice imaged $24 \mathrm{~h}$ after injection. (h) shows the unmixed PSS-794 signal for the mouse imaged $3 \mathrm{~h}$ post injection, and (k) the ICG signal also imaged $3 \mathrm{~h}$ post injection. The second column shows the corresponding epi-fluorescence images of cryo-slices through the tumor. Color photograph of cryo-slices are shown in the third column. The tumor area is delineated in yellow. 


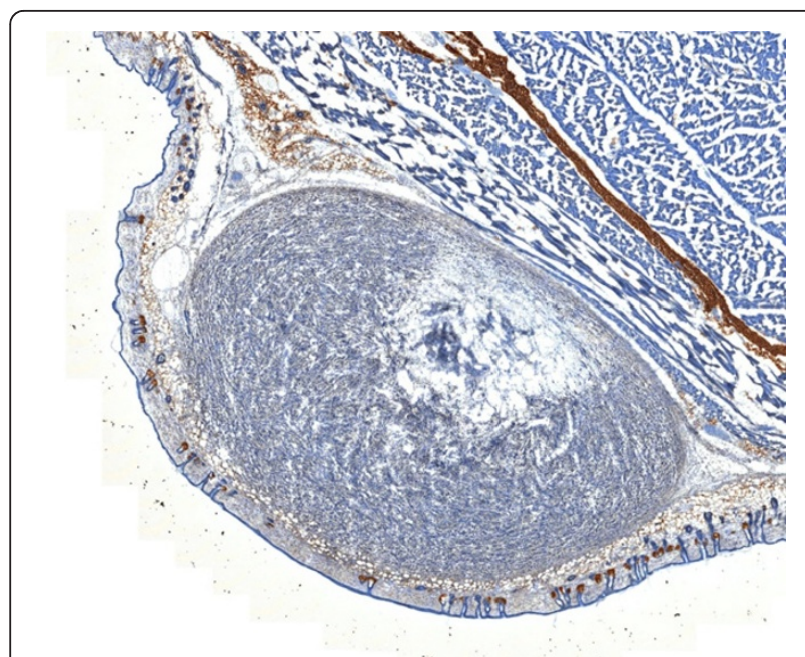

Figure $\mathbf{5}$ Representative histological slice of the tumor mass

the histological image in Figure 5 suggests that only a relatively small amount of apoptotic tissue exists in the tumor.

In summary, both the in vivo MSOT and the ex vivo epi-fluorescence images clearly reveal that the fluorescence signal of the PSS-794 probe is localized in the blood vessels surrounding the tumor. This observation agrees well with literature which shows that, once there is only a small amount of apoptotic tissue in this tumor cell line, PS-targeting probes will be only observed in the tumor blood vessels [31].

\section{Discussion}

Previous studies have shown that the fluorescent PS-targeting probe, PSS-794, can be used to optically image apoptotic/necrotic tissues often found inside tumors [22]. Here, it is demonstrated for the first time that PSS-794 also allows imaging of 4T1 tumor allografts without an actual infiltration of the probe into the tumor mass. In our experiments, lack of depth resolution of planar fluorescence imaging might have mistakenly led to the conclusion that PSS-794 is concentrated in the tumor mass. Even the use of three-dimensional optical tomography (FMT) has not attained the sufficient spatial resolution that would determine the precise location of the probe. In contrast, the much higher resolution of the MSOT revealed accumulation of the PSS794 in the blood vessels surrounding the tumor area and clearly showed no infiltration into the tumor mass. This lack of extravasation has been also confirmed by the epi-fluorescence images made on the cryo-sliced mouse. This suggests that the vasculature of the tumor was not leaky.

The lack of extravasation is not surprising in this case since both probes are known to associate with serum proteins, and it is hard for the large protein/dye complex to leak from the neovasculature, especially if there is a high interstitial pressure in the tumor [32]. On the other hand, since the unspecific ICG dye is not detected in the tumor area while PSS-794 stays in the tumor vessels much longer, we conclude that the PSS-794 is still targeting a certain biomarker most likely the PS exposed on vessel walls of the neovasculature $[33,34]$. There is evidence that $20-40 \%$ of the blood vessel surfaces in all tumors, and metastases that are larger than $1 \mathrm{~mm}$ expose PS even though these endothelial cells are vital and not apoptotic [35]. Moreover, it normally takes 6-24 $h$ to exert the enhanced permeability and retention (EPR) effect [36]. Thus, the strong retainment of PSS794 at the tumor by $3 \mathrm{~h}$ (Figure $3 \mathrm{~h}$ ) cannot be explained either by the EPR. This is because, if the tumors were leaky, both PSS-794 and ICG should have been observed within the tumor parenchyma.

The PS-binding antibody Bavituximab ${ }^{\circledR}$ (a human chimeric version of the murine monoclonal $\mathrm{IgG}_{3}$ antibody, 3G4) is especially effective at targeting this class of exposed PS, and it induces impressive antitumor activity in a wide array of tumors with no evidence of toxicity $[37,38]$. This raises the idea that zinc(II)-dipicolylmine complexes may have value as near-universal targeting agents for imaging and treatment of tumors; however, more studies are needed to test this interesting hypothesis. The current study showcases the power of MSOT to uncover new high resolution information about the precise location of imaging probes within sites of disease.

\section{Conclusions}

In conclusion, we used MSOT to accurately detect the targeted PSS-794 probe in vivo over strong background absorption of blood with spatial resolution on the order of $150 \mu \mathrm{m}$, attaining unprecedented image quality for deep-tissue imaging of optical contrast. The enhanced resolution of the MSOT clearly showed that the probe mainly localizes in the vessels surrounding the tumor, suggesting that the probe gains its tumor selectivity by targeting the PS that is exposed on the surface of the tumor blood vessels. The MSOT results further correlated well with cryo-slices and epi-fluorescence images of ex vivo specimens. Overall, this study demonstrates the high potential of MSOT to broadly impact the fields of tumor diagnostics and preclinical drug development.

\section{Acknowledgements}

VN acknowledges the support from the European Research Council (ERC) through an Advanced Investigator Award. DR acknowledges the support from the German Research Foundation (DFG) (grant no. RA 1848/1) and the ERC Starting Independent Researcher Grant. BDS acknowledges the NIH (USA). 


\section{Author details}

${ }^{1}$ Institute for Biological and Medical Imaging, Technische Universität München und Helmholtz Zentrum München, Ingoldstädter Landstraße 1 , Neuherberg 85764, Germany ${ }^{2}$ Notre Dame Integrated Imaging Facility, University of Notre Dame, Notre Dame, IN 46556-5670, USA

\section{Authors' contributions}

$A B, E H, V N$ and $D R$ designed the experiments. $A B$ and $E H$ performed the data analysis. AA did the FMT-XCT imaging. $A B, D R$ and $B D S$ have written the manuscript. BDS provided the PSS-794 contrast agent. DR and VN provided conceptual input and supervised the research. All authors read and approved the final manuscript.

\section{Competing interests}

The authors declare that they have no competing interests.

Received: 4 January 2012 Accepted: 1 April 2012 Published: 1 April 2012

\section{References}

1. Kiessling F, Razansky D, Alves F: Anatomical and microstructural imaging of angiogenesis. Eur J Nucl Med Mol Imaging 2010, 37(S1):S4-S19.

2. Weissleder R, Pittet MJ: Imaging in the era of molecular oncology. Nature 2008, 452:580-589

3. Ntziachristos $\mathrm{V}$, Tung $\mathrm{CH}$, Bremer $\mathrm{C}$, Weissleder R: Fluorescence molecular tomography resolves protease activity in vivo. Nat Med 2002, 8(7):757-760.

4. Gao XH, Yang L, Petros JA, Marshall FF, Simons JW, Nie S: In vivo molecular and cellular imaging with quantum dots. Curr Opin Biotechnol 2005, 16(1):63-72.

5. De La Zerda A, Zavaleta C, Keren S, Vaithilingam S, Bodapati S, Liu Z, Levi J, Smith BR, Ma TJ, Oralkan O, Cheng Z, Chen X, Dai H, Khuri-Yakub BT, Gambhir SS: Carbon nanotubes as photoacoustic molecular imaging agents in living mice. Nat Nanotechnol 2008, 3(9):557-562.

6. Peng XH, Qian X, Mao H, Wang AY, Chen Z, Nie S, Shin DM: Targeted magnetic iron oxide nanoparticles for tumor imaging and therapy. Int J Nanomedicine 2008, 3(3):311-321.

7. Ntziachristos $V$, Yodh AG, Schnall M, Chance B: Concurrent MRI and diffuse optical tomography of breast after indocyanine green enhancement. Proc Natl Acad Sci USA 2000, 97(6):2767-2772.

8. Valentini G, D'Andrea C, Ferrari R, Pifferi A, Cubeddu R, Martinelli M, Natoli C, Ubezio P, Giavazzi R: In vivo measurement of vascular modulation in experimental tumors using a fluorescent contrast agent. Photochem Photobiol 2008, 84(5):1249-1256.

9. Darzynkiewicz Z: Apoptosis in antitumor strategies: modulation of cell cycle or differentiation. J Cell Biochem 1995, 58(2):151-159.

10. Lennon SV, Martin SJ, Cotter TG: Dose-dependent induction of apoptosis in human tumour cell lines by widely diverging stimuli. Cell Prolif 1991, 24(2):203-214.

11. Edgington $L E$, Berger $A B$, Blum G, Albrow VE, Paulick MG, Lineberry N, Bogyo M: Noninvasive optical imaging of apoptosis by caspase-targeted activity-based probes. Nature Med 2009, 15(8):967-U177.

12. Petrovsky A, Schellenberger E, Josephson L, Weissleder R, Bogdanov A: Near-infrared fluorescent imaging of tumor apoptosis. Cancer Res 2003, 63(8):1936-1942.

13. Ntziachristos $V$, Schellenberger EA, Ripoll J, Yessayan D, Graves E, Bogdanov A, Josephson L, Weissleder R: Visualization of antitumor treatment by means of fluorescence molecular tomography with an Annexin V-Cy5.5 conjugate. Proc Natl Acad Sci USA 2004, 101(33):12294-12299.

14. Sosnovik DE, Nahrendorf M, Deliolanis N, Novikov M, Aikawa E, Josephson L, Rosenzweig A, Weissleder R, Ntziachristos V: Fluorescence tomography and magnetic resonance imaging of myocardial macrophage infiltration in infarcted myocardium in vivo. Circulation 2007, 115(11):1384-1391.

15. Ntziachristos $V$, Razansky D: Molecular imaging by means of multispectral optoacoustic tomography (MSOT). Chem Rev 2010, 110(5):2783-2794.

16. Wang LV: Prospects of photoacoustic tomography. Med Physics 2008, 35(12):5758-5767.

17. Razansky D, Distel M, Vinegoni C, Ma R, Perrimon N, Köster RW, Ntziachristos V: Multispectral opto-acoustic tomography of deep-seated fluorescent proteins in vivo. Nat Photonics 2009, 3(7):412-417.
18. Razansky D, Vinegoni C, Ntziachristos V: Multispectral photoacoustic imaging of fluorochromes in small animals. Opt Lett 2007, 32:2891-2893.

19. Buehler A, Herzog E, Razansky D, Ntziachristos V: Video rate optoacoustic tomography of mouse kidney perfusion. Opt Lett 2010, 35(14):2475-2477.

20. Gamelin J, Maurudis A, Aguirre A, Huang F, Guo P, Wang LV, Zhu Q: A realtime photoacoustic tomography system for small animals. Opt Express 2009, 17(13):10489-10498.

21. Razansky D, Buehler A, Ntziachristos V: Volumetric real-time multispectral optoacoustic tomography of biomarkers. Nat Protoc 2011, 6(8):1121-1129.

22. Smith BA, Akers WJ, Leevy WM, Lampkins AJ, Xiao S, Wolter W, Suckow MA, Achilefu S, Smith BD: Optical imaging of mammary and prostate tumors in living animals using a synthetic near infrared zinc(II)-dipicolylamine probe for anionic cell surfaces. J Am Chem Soc 2010, 132(1):67-69.

23. Evan G, Littlewood T: A matter of life and cell death. Science 1998 281(5381):1317-1322.

24. Leevy WM, Gammon ST, Jiang H, Johnson JR, Maxwell DJ, Jackson EN, Marquez M, Piwnica-Worms D, Smith BD: Optical imaging of bacterial infection in living mice using a fluorescent near-infrared molecular probe. J Am Chem Soc 2006, 128(51):16476-16477.

25. Rosenthal A, Razansky D, Ntziachristos V: Fast semi-analytical model-based acoustic inversion for quantitative optoacoustic tomography. IEEE Trans Med Imaging 2010, 28(12):1997-2006.

26. Rosenthal A, Ntziachristos $V$, Razansky D: Optoacoustic methods for frequency calibration of ultrasonic sensors. IEEE Trans Ultrason Ferroelectr Frea Control 2011, 58(2):316-326.

27. Buehler A, Rosenthal A, Jetzfellner T, Dima A, Razansky D, Ntziachristos V: Model-based optoacoustic inversions with incomplete projection data. Med Physics 2011, 38(3):1694-1704.

28. Glatz J, Deliolanis NC, Buehler A, Razansky D, Ntziachristos V: Blind source unmixing in multi-spectral optoacoustic tomography. Opt Express 2011, 19(4):3175-3184.

29. Schulz RB, Ale A, Sarantopoulos A, Freyer M, Soehngen E, Zientkowska M, Ntziachristos $\mathrm{V}$ : Hybrid system for simultaneous fluorescence and X-ray computed tomography. IEEE Trans Med Imaging 2010, 29(2):465-473.

30. Sarantopoulos A, Themelis G, Ntziachristos V: Imaging the bio-distribution of fluorescent probes using multispectral epi-illumination cryoslicing imaging. Mol Imaging Biol 2011, 13(5):874-885.

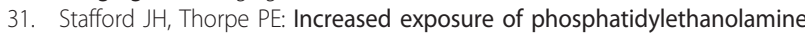
on the surface of tumor vascular endothelium. Neoplasia 2011, 13(4):299-308.

32. Berezin MY, Guo K, Akers W, Livingston J, Solomon M, Lee H, Liang K, Agee A, Achilefu S: Rational approach to select small peptide molecular probes labeled with fluorescent cyanine dyes for in vivo optical imaging. Biochemistry 2011, 50(13):2691-2700.

33. Kenis $\mathrm{H}$, Reutelingsperger $\mathrm{C}$ : Targeting phosphatidylserine in anti-cancer therapy. Curr Pharm Des 2009, 15(23):2719-2723.

34. Thorpe PE: Targeting anionic phospholipids on tumor blood vessels and tumor cells. Thromb Res 2010, 125:S134-S137.

35. Ran S, Downes A, Thorpe PE: Increased exposure of anionic phospholipids on the surface of tumor blood vessels. Cancer Res 2002, 62(21):6132-6140.

36. Maeda $\mathrm{H}$ : The enhanced permeability and retention (EPR) effect in tumor vasculature: the key role of tumor-selective macromolecular drug targeting. Adv Enzym Regul 2001, 41:189-207.

37. Huang XM, Bennett $M$, Thorpe PE: A monoclonal antibody that binds anionic phospholipids on tumor blood vessels enhances the antitumor effect of docetaxel on human breast tumors in mice. Cancer Res 2005, 65(10):4408-4416.

38. Ran S, Jin He, Huang X, Soares M, Scothorn D, Thorpe PE: Antitumor effects of a monoclonal antibody that binds anionic phospholipids on the surface of tumor blood vessels in mice. Clin Cancer Res 2005, 11(4):1551-1562

\section{doi:10.1186/2191-219X-2-14}

Cite this article as: Buehler et al:: High resolution tumor targeting in living mice by means of multispectral optoacoustic tomography. EJNMMI Research 2012 2:14. 